\title{
Knockdown of $\operatorname{lncRNA} M E G 8$ inhibits cell proliferation and invasion, but promotes cell apoptosis in hemangioma, via miR-203-induced mediation of the Notch signaling pathway
}

\author{
ZHENFENG HU ${ }^{1}$, XIANGMEI LIU ${ }^{2}$, JING GUO $^{3}$, LEI ZHUO $^{1}$, YONGDONG CHEN $^{4}$ and HAOJUN YUAN ${ }^{1}$ \\ ${ }^{1}$ Department of General Surgery II (Department of Plastic Surgery), Affiliated Hospital of Hebei University of Engineering, \\ Hebei, Handan 056002; ${ }^{2}$ Department of Plastic Surgery, Handan Seventh Hospital; ${ }^{3}$ Department of Cardiology, \\ Handan Central Hospital, Hebei, Handan 056001; ${ }^{4}$ Department of General Surgery III, \\ Handan First Hospital, Hebei, Handan 056002, P.R. China
}

Received March 16, 2021; Accepted July 20, 2021

DOI: $10.3892 / \mathrm{mmr} .2021 .12512$

\begin{abstract}
As a member of the long non-coding (lnc)RNA family, lncRNA maternally expressed 8 , small nucleolar RNA host gene (MEG8), has been reported to serve an oncogenic role in several types of malignancies, including hepatocellular carcinoma, non-small cell lung cancer and pancreatic cancer. The current study aimed to investigate the effect of the knockdown of $M E G 8$ on human hemangioma endothelial cell (HemEC) proliferation, apoptosis and invasion, in addition to determining the underlying molecular mechanism. The knockdown of lncRNA MEG8 was achieved by transfecting lncRNA MEG8 small interfering (si)RNA into HemECs, while the combined knockdown of lncRNA MEG8 knockdown and microRNA (miR)-203 was established by co-transfecting lncRNA MEG8 siRNA and a miR-203 inhibitor into HemECs. The cell proliferation, apoptosis and invasion and the expression levels of miR-34a, miR-200b, miR-200b and Notch signaling pathway-related factors were detected via CCK-8 Kit, flow cytometry, Transwell, reverse transcription-quantitative PCR and western blot assay, respectively. The knockdown of lncRNA MEG8 significantly inhibited proliferation $(\mathrm{P}<0.05)$ and invasion $(\mathrm{P}<0.05)$, but promoted apoptosis $(\mathrm{P}<0.01)$ in HemECs. Furthermore, IncRNA MEG8 knockdown upregulated $m i R-203(\mathrm{P}<0.01)$ expression, but did not alter $m i R-34 a$ or $m i R-200 b$ expression (both $\mathrm{P}>0.05$ ). Subsequent experiments revealed that $m i R$-203 silencing exerted no significant effect on the expression levels of lncRNA MEG8 $(\mathrm{P}>0.05)$ in HemECs. In addition, $m i R-203$ silencing increased cell
\end{abstract}

Correspondence to: Dr Xiangmei Liu, Department of Plastic Surgery, Handan Seventh Hospital, 33 Xuechi Road, Hebei, Handan 056001, P.R. China

E-mail: xianglandao7332037@163.com

Key words: hemangioma, long non-coding RNA maternally expressed 8, small nucleolar RNA host gene, microRNA-203, Notch signaling pathway, proliferation, invasion proliferation $(\mathrm{P}<0.05)$ and invasion $(\mathrm{P}<0.01)$, but suppressed apoptosis $(\mathrm{P}<0.05)$. miR-203 silencing also reversed the effect of $\operatorname{lncRNA}$ MEG8 knockdown on the proliferation $(\mathrm{P}<0.05)$, apoptosis $(\mathrm{P}<0.001)$ and invasion $(\mathrm{P}<0.01)$ of HemECs. Moreover, lncRNA MEG8 knockdown downregulated jagged canonical notch ligand $1(\mathrm{JAGl} ; \mathrm{P}<0.05)$ and Notch1 $(\mathrm{P}<0.05)$ expression levels, while $m i R-203$ silencing upregulated JAG1 $(\mathrm{P}<0.01)$ and Notch1 $(\mathrm{P}<0.01)$ expression levels and reversed the effects of $\operatorname{lncRNA} M E G 8$ knockdown on JAG1 $(\mathrm{P}<0.01)$ and Notch1 $(\mathrm{P}<0.01)$ expression in HemECs. In conclusion, the findings of the present study suggested that $\operatorname{lncRNA} M E G 8$ knockdown may inhibit cell proliferation and invasion, but promote cell apoptosis in hemangioma via $m i R$-203-induced mediation of the Notch signaling pathway.

\section{Introduction}

A hemangioma is a common benign vascular tumor that often occurs in childhood and more frequently affects females (1). Hemangiomas typically follow a characteristic pattern of a proliferative growth phase, followed by a slow involuting phase, during which the majority of hemangiomas stop growing and begin to shrink (2). During the proliferative growth phase, hemangiomas present with several symptomatic clinical manifestations, including ulcerations, bleeding, physical functional limitations and breakdown in the surface of the skin, and current treatment options comprise medical therapy, laser therapy and surgical resection $(2,3)$. However, cases of severe hemangioma are challenging to treat in the clinic. Hence, further understanding of the mechanisms underlying hemangioma progression is required.

Long non-coding (lnc) RNAs are a group of non-coding transcripts of $>200$ nucleotides in length, which comprise 4-9\% of mammalian transcriptomes (4). IncRNAs have been discovered to be involved in numerous physiological and pathobiological processes. For example, previous studies report that the expression levels of lncRNAs are dysregulated and thereby implicated in several processes during malignancy, including malignant cell proliferation, invasion, migration, epithelial-mesenchymal transition (EMT) and drug resistance (5-7). 
Notably, lncRNA maternally expressed 8, small nucleolar RNA host gene (MEG8), as a member of the lncRNA family, has been found to serve an oncogenic role in several types of malignancy, including hepatocellular carcinoma (HCC), non-small cell lung cancer (NSCLC) and pancreatic cancer (8-11). For instance, $\operatorname{lncRNA} M E G 8$ promotes NSCLC cell proliferation, migration and invasion via targeting the microRNA (miRNA/miR)-107/CDK6 axis (9). In addition, lncRNA MEG8 targets several miRNAs ( $m i R-34 a / m i R-200 b / m i R-203)$ to promote cell morphological changes and enhance cell motility in tumors (10). In another study, $\operatorname{lncRNA} M E G 8$ regulates the Notch signaling pathway to mediate the EMT of hepatic stellate cells, thereby serving a role in the pathological processes of liver fibrosis as well as HCC (12). Of note, the Notch signaling pathway is a conserved ligand-receptor signaling pathway that modulates cell-cell interactions and the activity of VEGF, which has been shown to serve a role in the pathogenesis of hemangioma (13). Therefore, it was hypothesized that $\operatorname{lncRNAMEG8}$ may be involved in the pathological process of hemangiomas via targeting possible miRNAs (miR-34a/miR-200b/miR-203) and Notch signaling pathway; however, the underlying mechanism remains to be elucidated.

The current study aimed to investigate the effect of lncRNA MEG8 knockdown on cell proliferation, apoptosis and invasion, as well as to determine its possible molecular mechanism in hemangioma.

\section{Materials and methods}

Cell lines and culture. Human hemangioma endothelial cells (HemECs) were isolated from 3 individuals ( 2 females and 1 male, mean age of 14 months with range 9-24 months) with proliferating-phase hemangioma as previously described (14). The HemECs were cultured in Human Endothelial Serum Free medium (Gibco; Thermo Fisher Scientific, Inc.) supplemented with 10\% FBS (Gibco; Thermo Fisher Scientific, Inc.) and maintained in a humidified incubator with $5 \% \mathrm{CO}_{2}$ at $37^{\circ} \mathrm{C}$. The experimental protocol was approved by the Ethics Committee of Handan Seventh Hospital (approval no. 2019[K]016) and written informed consent was obtained from the parents/guardians of each patient prior to participation.

Cell transfection. IncRNA MEG8 small interfering RNA (siRNA), negative control (NC) siRNA and $m i R-203$ inhibitor were purchased from Shanghai GenePharma Co., Ltd. The sequences for lncRNA MEG8 siRNA, NC siRNA, miR-203 inhibitor and NC inhibitor were as follows: lncRNA MEG8 sense, 5'GGCCAGCUGAUUUAAUAAUUU3', lncRNA MEG8 antisense, 5'AUUAUUAAAUCAGCUGGCCUU3'; NC siRNA sense, 5'GAAUUAAUUAAAGAUGGCCCGUUG UAC3', NC siRNA antisense, 5'UCAUCGAAGUUAUAG GGAUACAUUACGUGAUC3'; miR-203 inhibitor, 5'CUA GUGGUCCUAAACAUUUCAC3'; NC inhibitor, 5'CAGUAC UUUUGUGUAGUACAA3'. A total of $2 \times 10^{5}$ HemECs were seeded into 6 -well plates $24 \mathrm{~h}$ prior to transfection. Following incubation, the HemECs were transfected with $50 \mathrm{pM} \mathrm{NC}$ siRNA, 50 pM lncRNA MEG8 siRNA, 50 pM NC inhibitor, $50 \mathrm{pM}$ miR-203 inhibitor or $50 \mathrm{pM}$ lncRNA MEG8 siRNA + 50 pM miR-203 inhibitor using Lipofectamine ${ }^{\circledR} 2000$ reagent (Invitrogen; Thermo Fisher Scientific, Inc.) for $6 \mathrm{~h}$ at $37^{\circ} \mathrm{C}$, according to the manufacturer's protocol. Untransfected HemECs were used as the control.

miR-203 transfection efficiency assessment. NC inhibitor, NC mimic and $m i R-203$ mimic were purchased from Shanghai GenePharma Co. The NC mimic, miR-203 mimic, NC inhibitor and $m i R-203$ inhibitor were transfected into HemECs with the application of Lipofectamine 2000 reagent as aforementioned. The HemECs without transfection were served as normal control. The transfection efficacy was validated by detecting $m i R-203$ expression at $24 \mathrm{~h}$ post transfection, as shown in Fig. S1.

Reverse transcription-quantitative $(R T-q) P C R$. At $24 \mathrm{~h}$ post-transfection, $2 \times 10^{6}$ HemECs were harvested and underwent RT-qPCR. Briefly, total RNA was extracted from cells using a RNeasy Mini kit (Qiagen $\mathrm{GmbH}$ ) and reverse transcribed into cDNA using a cDNA Reverse Transcription kit (Applied Biosystems; Thermo Fisher Scientific, Inc.) according to the kit's instructions. qPCR was subsequently performed using a SYBR ${ }^{\mathrm{TM}}$ Green PCR Master mix (Applied Biosystems; Thermo Fisher Scientific, Inc.) by following the kit's protocol. The following thermocycling conditions were used for the qPCR: Initial denaturation at $95^{\circ} \mathrm{C}$ for $10 \mathrm{~min}$; followed by 40 cycles of $95^{\circ} \mathrm{C}$ for $15 \mathrm{sec}$ and $61^{\circ} \mathrm{C}$ for $1 \mathrm{~min}$. LncRNA (lncRNA MEG8) and mRNA [jagged canonical notch ligand 1 (JAGl), NOTCHI] were normalized to $\beta$-actin and miRNAs ( $m i R-34 a, m i R-200 b, m i R-203)$ were normalized to $U 6$. The experiments were repeated 3 times. These gene expression levels were quantified using the $2^{-\Delta \Delta \mathrm{Cq}}$ method (15). The primers used for the qPCR are listed in Table I.

Cell Counting Kit-8 (CCK-8) assay. Cell proliferation was analyzed using a CCK-8 assay (Sigma-Aldrich; Merck KGaA) at $0,24,48$ or $72 \mathrm{~h}$ post-transfection. Briefly, CCK-8 reagent was added to the cells at each time point and incubated for a further $2 \mathrm{~h}$. The optical density value was measured using a microplate reader (BioTek Instruments, Inc.) at $450 \mathrm{~nm}$.

Flow cytometric analysis of cell apoptosis. HemECs were harvested for apoptosis analysis at $48 \mathrm{~h}$ post-transfection. Briefly, HemECs were centrifuged $\left(800 \mathrm{~g}, 5 \mathrm{~min}, 4^{\circ} \mathrm{C}\right)$ and resuspended, then incubated with Annexin V-FITC (eBioscience; Thermo Fisher Scientific, Inc.) and PI (eBioscience; Thermo Fisher Scientific, Inc.) in the dark for $20 \mathrm{~min}$ at room temperature. Apoptotic HemECs were analyzed using a flow cytometer (FACSCalibur; BD Biosciences) and a FlowJo 7.6 software (BD Biosciences). The apoptosis rate was defined as percentage of early plus late apoptotic cells.

Transwell assay. The invasive ability of HemECs was analyzed using a Transwell assay. At $24 \mathrm{~h}$ post-transfection, cells were collected and seeded into Matrigel basement membrane matrix-coated Transwell inserts (Corning, Inc.). The medium in upper and lower chambers was Human Endothelial Serum Free medium (Gibco; Thermo Fisher Scientific, Inc.) and 10\% FBS (Gibco; Thermo Fisher Scientific, Inc.), respectively. After incubation for another $24 \mathrm{~h}$, the cells remaining in the top of the inserts were removed and the cells in the bottom were fixed with $10 \%$ formalin (Sigma-Aldrich; Merck KGaA) for $15 \mathrm{~min}$ at room temperature and stained with crystal 
Table I. Primers.

\begin{tabular}{lll}
\hline Gene & \multicolumn{1}{c}{ Forward $\left(5^{\prime} \rightarrow 3^{\prime}\right)$} & \multicolumn{1}{c}{ Reverse $\left(5^{\prime} \rightarrow 3^{\prime}\right)$} \\
\hline lncRNA $M E G 8$ & GCCACCAGCCTTATGATTGC & TCCTAACACAGAGAACCAACCAT \\
$J A G l$ & TGGTTAATGGTTATCGCTGTATCTG & ATAGTCACTGGCACGGTTGTAG \\
NOTCH1 & CAGAGGCGTGGCAGACTATG & GGCAGTGGCAGATGTAGGAG \\
- actin & TCGTGCGTGACATTAAGGAGAAG & AGGACTCCATGCCCAGGAA \\
$m i R-34 a$ & ACACTCCAGCTGGGTGGCAGTGTCTTAGCT & TGTCGTGGAGTCGGCAATTC \\
$m i R-200 b$ & ACACTCCAGCTGGGCATCTTACTGGGCAGC & TGTCGTGGAGTCGGCAATTC \\
$m i R-203$ & ACACTCCAGCTGGGGTGAAATGTTTAGGAC & TGTCGTGGAGTCGGCAATTC \\
$U 6$ & GCTCGCTTCGGCAGCACATA & AATATGGAACGCTTCACGAATTTGC
\end{tabular}

Table II. Antibodies.

Antibody

Company

Dilution

\author{
Primary antibodies \\ Rabbit polyclonal to Cleaved caspase-3 \\ Rabbit monoclonal to Cleaved caspase-7 \\ Rabbit polyclonal to JAG1 \\ Rabbit polyclonal to NOTCH1 \\ Rabbit monoclonal to $\beta$-actin \\ Secondary antibody \\ Goat Anti-Rabbit IgG H\&L (HRP)
}

Abcam

$1: 500$

Abcam

$1: 1,000$

Invitrogen; Thermo Fisher Scientific, Inc.

$1: 1,000$

Invitrogen; Thermo Fisher Scientific, Inc.

$1: 2,000$

Invitrogen; Thermo Fisher Scientific, Inc.

$1: 2,000$

Invitrogen; Thermo Fisher Scientific, Inc.

$1: 50,000$

violet (Sigma-Aldrich; Merck $\mathrm{KGaA}$ ) for $20 \mathrm{~min}$ at room temperature. Invasive cells were observed using an inverted microscope (Nikon Corporation) at the magnification of $\mathrm{x} 200$, with 5 fields being chosen randomly.

Western blotting. Total protein was extracted from HemECs at $48 \mathrm{~h}$ post-transfection using RIPA lysis buffer (Sigma-Aldrich; Merck KGaA). Total protein was quantified using a BCA Protein assay kit (Thermo Fisher Scientific, Inc.) and $20 \mu \mathrm{g}$ protein/lane was separated using 4-20\% precast polyacrylamide gels (Sigma-Aldrich; Merck KGaA). The separated proteins were subsequently transferred onto nitrocellulose filter membranes (Pall Life Sciences) and blocked with 5\% BSA (Sigma-Aldrich; Merck KGaA) for $1.5 \mathrm{~h}$. The membranes were then incubated with primary antibodies at $4^{\circ} \mathrm{C}$ overnight. Following the primary antibody incubation, the membranes were incubated with a secondary antibody for $1.5 \mathrm{~h}$. Protein bands were visualized using ECL western blotting substrate (Thermo Fisher Scientific, Inc.). The protein density was evaluated with ImageJ (Version 1.8.0, National Institutes of Health). The antibodies used for western blotting are listed in Table II.

Dual luciferase reporter gene assay. The wild-type (WT) or mutant type (MT) lncRNA MEG8 sequences were cloned into a pGLuc plasmid (Beyotime Institute of Biotechnology) to generate WT or MT plasmids. NC mimic or miR-203 mimic were co-transfected with WT or MT plasmid into HemECs with the application of Lipofectamine ${ }^{\circledR} 2000$ reagent (Invitrogen; Thermo Fisher Scientific, Inc.). Following $48 \mathrm{~h}$ of transfection, the cells were harvested and the relative luciferase activity was measured using a Dual Luciferase Reporter Gene assay kit (Beyotime Institute of Biotechnology).

Statistical analysis. Statistical analysis was performed using GraphPad Prism 7.02 software (GraphPad Software, Inc.) and presented as the mean \pm standard deviation. Statistical differences between two groups were determined using an unpaired Student's t-test, while the comparisons among multiple groups were performed using a one-way ANOVA followed by a Tukey's post hoc test. $\mathrm{P}<0.05$ was considered to indicate a statistically significant difference.

\section{Results}

Effect of lncRNA MEG8 knockdown on the proliferation, apoptosis and invasion of HemECs. The relative expression levels of $\operatorname{lncRNA} M E G 8$ were downregulated following the transfection with $\operatorname{lncRNA} M E G 8$ siRNA $(\mathrm{P}<0.001)$, which suggested the successful transfection of the siRNA (Fig. 1A). In addition, IncRNA MEG8 siRNA also decreased cell proliferation following $48(\mathrm{P}<0.05)$ or $72 \mathrm{~h}(\mathrm{P}<0.05)$ of transfection (Fig. 1B). Conversely, cell apoptosis was increased by transfection with lncRNA MEG8 siRNA ( $\mathrm{P}<0.01$; Fig. 1C-E). Furthermore, the number of invasive cells was decreased following the transfection with lncRNA MEG8 siRNA ( $\mathrm{P}<0.05$; Fig. 2A and $\mathrm{B})$.

Effect of lncRNA MEG8 knockdown on miR-34a, miR-200b and miR-203 expression levels in HemECs. Transfection with lncRNA MEG8 siRNA exerted no significant effect on $m i R-34 a(\mathrm{P}>0.05$; Fig. 3A) and $m i R-200 b$ ( $\mathrm{P}>0.05$; Fig. 3B) 
A

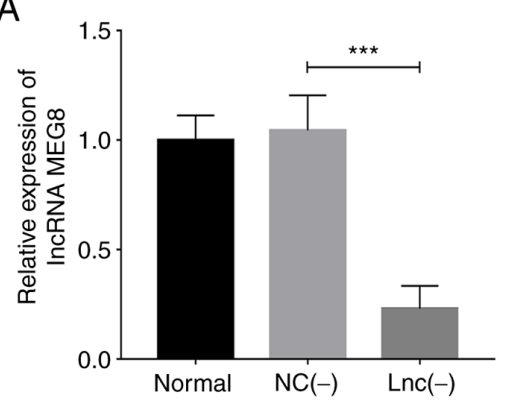

B

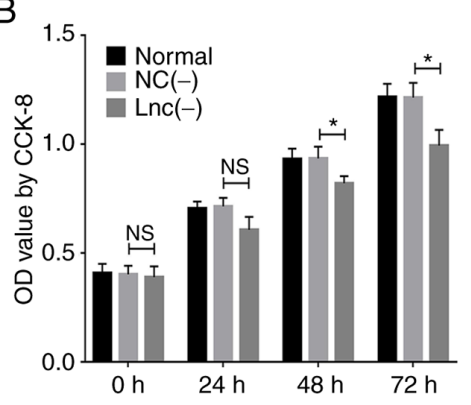

C

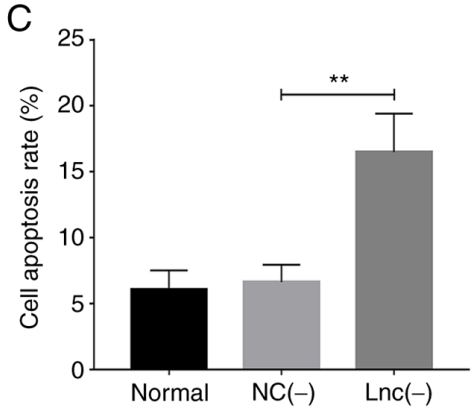

D

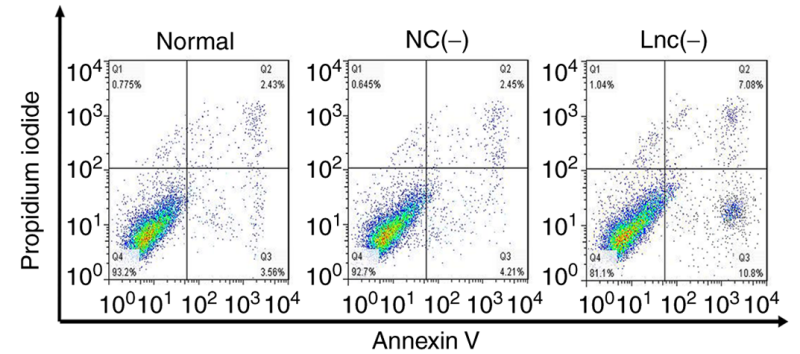

E

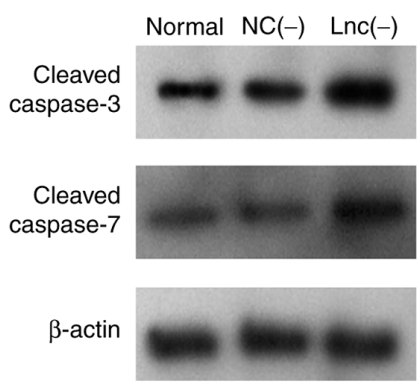

Figure 1. IncRNA MEG8 knockdown inhibits the proliferation but promotes the apoptosis of hemangioma endothelial cells. (A) Analysis of $\operatorname{lncRNA} M E G 8$ expression levels, (B) cell proliferation, (C and D) cell apoptosis and (E) cleaved caspase-3 and caspase-7 protein expression levels in the NC(-) and lnc(-) groups. Statistical differences among groups were determined using a one-way ANOVA followed by a Tukey's post hoc test. All experiments were conducted in triplicate. ${ }^{*} \mathrm{P}<0.05,{ }^{* *} \mathrm{P}<0.01,{ }^{* * *} \mathrm{P}<0.001$. IncRNA, long non-coding RNA; MEG8, maternally expressed 8, small nucleolar RNA host gene; NC, negative control; lnc (-), cells transfected with IncRNA MEG8 small interfering RNA; OD, optical density.

A

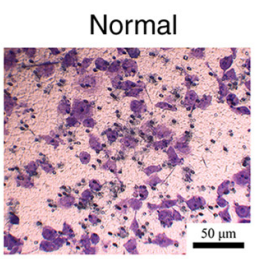

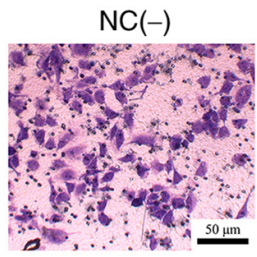
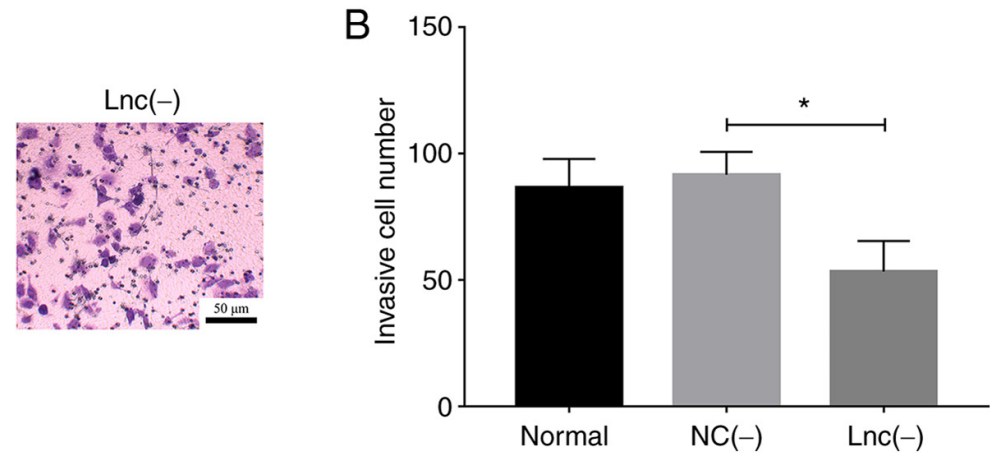

Figure 2. Knockdown of IncRNA MEG8 suppresses the invasion of hemangioma endothelial cells. (A and B) Cell invasion was measured in the NC (-) and lnc (-) groups. Statistical differences among groups were determined using a one-way ANOVA followed by a Tukey's post hoc test. All experiments were conducted in triplicate. "P<0.05. IncRNA, long non-coding RNA; MEG8, maternally expressed 8, small nucleolar RNA host gene; NC, negative control; lnc (-), cells transfected with IncRNA MEG8 small interfering RNA; NS, not significant.

expression levels; however, $m i R$-203 expression levels were upregulated $(\mathrm{P}<0.01$; Fig. $3 \mathrm{C})$.

Association between IncRNA MEG8 and miR-203 in HemECs. The binding site between WT IncRNA MEG 8 and $m i R-203$ is shown in Fig. 4A. The results of the dual luciferase reporter gene assay indicated that the knockdown of $m i R-203$ decreased the relative luciferase activity of the IncRNA MEG8 WT vector $(\mathrm{P}<0.01)$, but did not affect the relative luciferase activity of the lncRNA MEG8 MT vector ( $\mathrm{P}>0.05$; Fig. 4B). Transfection with the $m i R-203$ inhibitor did not significantly alter IncRNA MEG8 expression levels ( $\mathrm{P}>0.05$; Fig. $4 \mathrm{C})$, whereas the transfection with IncRNA MEG8 siRNA upregulated miR-203 expression levels $(\mathrm{P}<0.001$; Fig. 4D). These findings indicated that IncRNA $M E G 8$ may negatively regulate $m i R-203$ expression.
Knockdown oflncRNAMEG8regulates miR-203-induced regulation of proliferation and apoptosis in HemECs. Transfection with the $m i R-203$ inhibitor increased cell proliferation at 48 $(\mathrm{P}<0.05)$ and $72 \mathrm{~h}(\mathrm{P}<0.05)$, whereas the co-transfection with the miR-203 inhibitor reversed the effect of $\operatorname{lncRNA} M E G 8$ siRNA on cell proliferation at $48(\mathrm{P}<0.05)$ and $72 \mathrm{~h}(\mathrm{P}<0.05$; Fig. 5A). Furthermore, transfection with the miR-203 inhibitor suppressed cell apoptosis $(\mathrm{P}<0.001)$ and reversed the effect of lncRNA MEG8 siRNA on cell apoptosis ( $\mathrm{P}<0.001$; Fig. 5B-D).

Knockdown of IncRNA MEG8 regulates the miR-203-induced regulation of invasion in HemECs. Transfection with the $m i R-203$ inhibitor promoted cell invasion $(\mathrm{P}<0.01)$ and reversed the effect of IncRNA MEG8 siRNA on cell invasion $(\mathrm{P}<0.01$; Fig. 6A and B). 

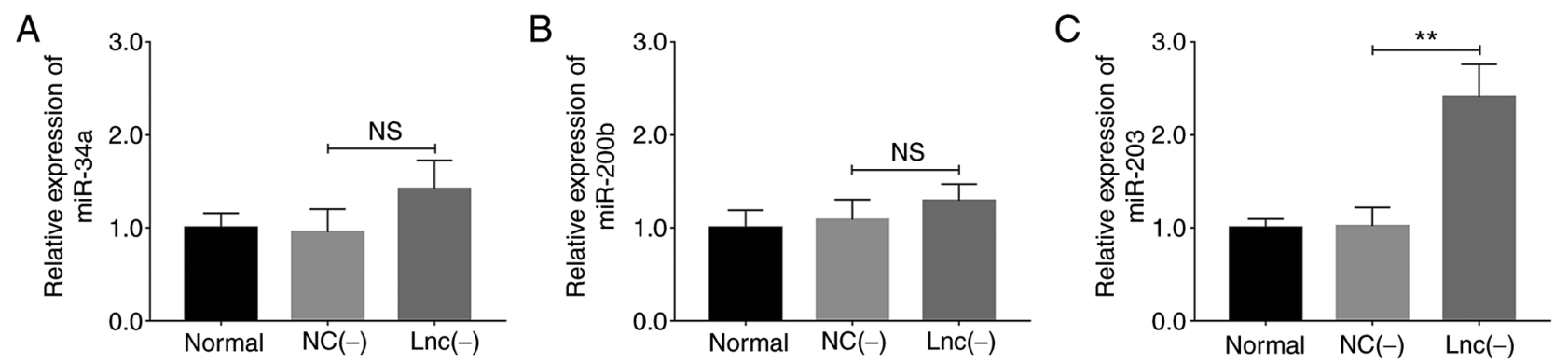

Figure 3. IncRNA MEG8 knockdown upregulates $m i R-203$, but not $m i R-34 a$ and $m i R-200 b$ expression levels in hemangioma endothelial cells. Analysis of (A) $m i R-34 a$, (B) $m i R-200 b$ and (C) $m i R-203$ expression levels in the NC (-) and lnc (-) groups. Statistical differences among groups were determined using a one-way ANOVA followed by a Tukey's post hoc test. All experiments were conducted in triplicate. ${ }^{* *} \mathrm{P}<0.01$. IncRNA, long non-coding RNA; $M E G 8$, maternally expressed 8, small nucleolar RNA host gene; NC, negative control; lnc (-), cells transfected with lncRNA MEG8 small interfering RNA; miR, microRNA; NS, not significant.

A
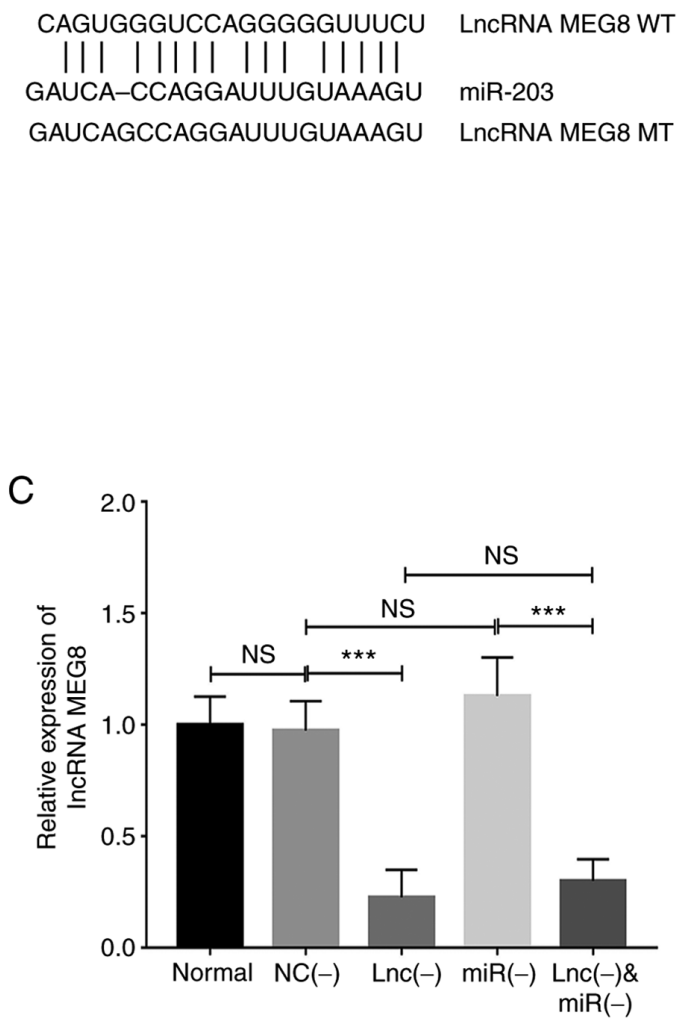

B
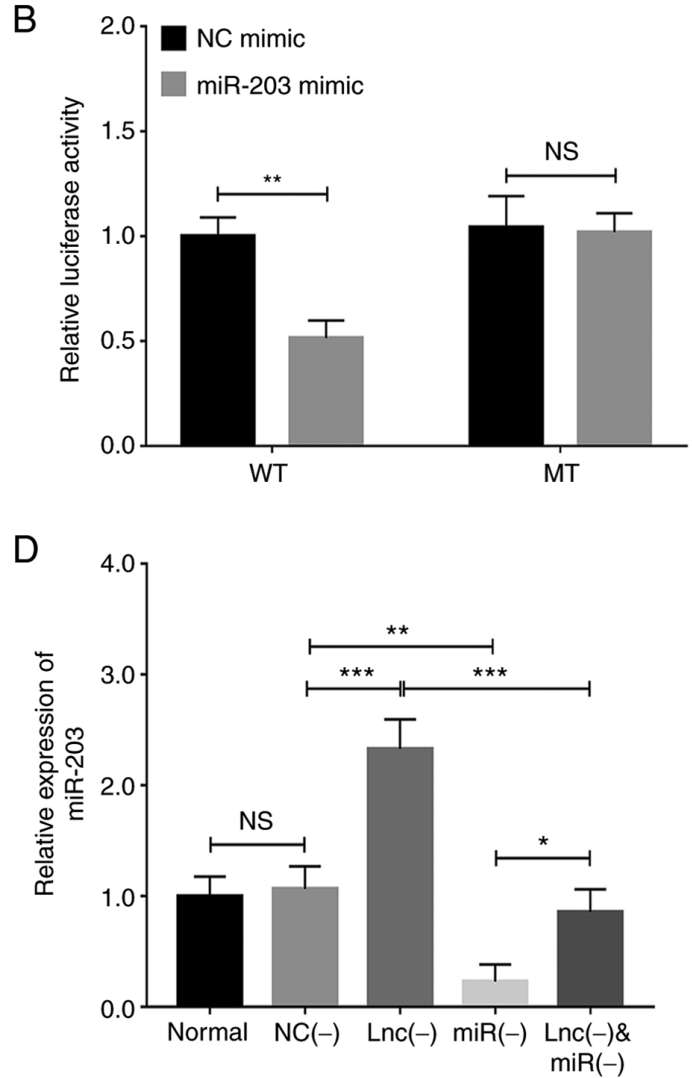

Figure 4. IncRNA MEG8 knockdown upregulates miR-203 expression in hemangioma endothelial cells. (A) Binding sites between WT lncRNA MEG8 and $m i R-203$ are shown. (B) Relative luciferase activity in cells co-transfected with $m i R-203$ mimic or NC mimic and WT or MT vectors. (C) lncRNA MEG8 and (D) $m i R-203$ expression levels were analyzed in the normal, NC (-), lnc (-), miR (-) and lnc (-) + miR (-) groups. Statistical differences between two groups were determined using an unpaired Student's t-test, while statistical differences between multiple groups were determined using a one-way ANOVA followed by a Tukey's post hoc test. All experiments were conducted in triplicate. ${ }^{*} \mathrm{P}<0.05,{ }^{* *} \mathrm{P}<0.01,{ }^{* * * *} \mathrm{P}<0.001$. IncRNA, long non-coding RNA; MEG8, maternally expressed 8, small nucleolar RNA host gene; miR, microRNA; WT, wild-type; MT, mutant type; NC, negative control; Inc (-), cells transfected with lncRNA $M E G 8$ small interfering RNA; miR (-), cells transfected with the miR-203 inhibitor; NS, not significant.

Knockdown of IncRNA MEG8 regulates the miR-203-induced mediation of the Notch signaling pathway in HemECs. Transfection with lncRNA MEG8 siRNA downregulated $J A G 1$ mRNA expression levels $(\mathrm{P}<0.05)$, while the miR-203 inhibitor upregulated JAG1 mRNA expression levels $(\mathrm{P}<0.01)$ and reversed the effect of lncRNA MEG8 siRNA on JAGl mRNA expression in HemECs $(\mathrm{P}<0.01$; Fig. 7A). Moreover, transfection with $\ln c R N A M E G 8$ siRNA downregulated Notchl mRNA expression levels $(\mathrm{P}<0.05)$; however, the
miR-203 inhibitor upregulated the mRNA expression levels of Notch1 $(\mathrm{P}<0.01)$ and reversed the effect of lncRNA MEG8 siRNA on NotchlmRNA expression ( $\mathrm{P}<0.01$; Fig. 7B). These data were further validated by western blotting (Fig. 7C).

\section{Discussion}

lncRNA MEG8 has been reported to serve a role in the tumorigenesis of several tumor types (8-12). For example, 
A

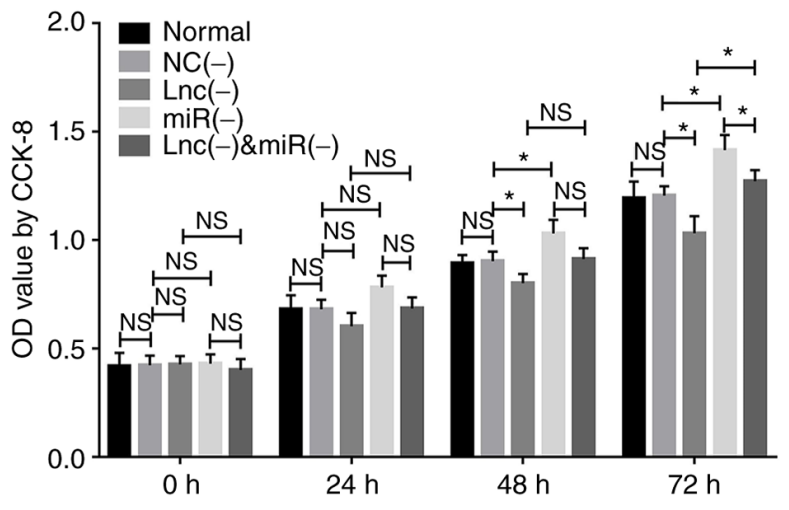

C

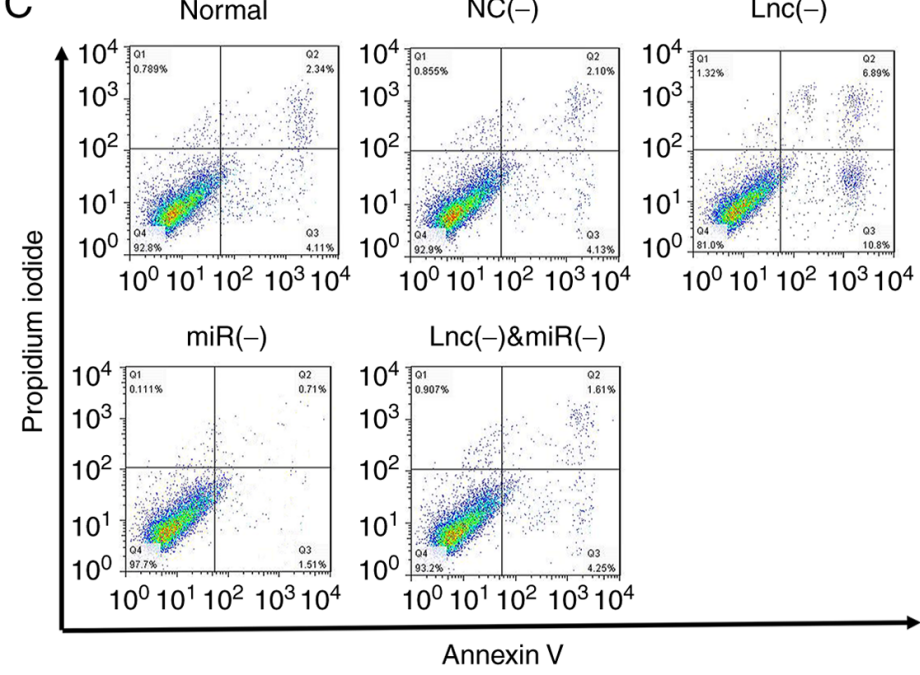

B

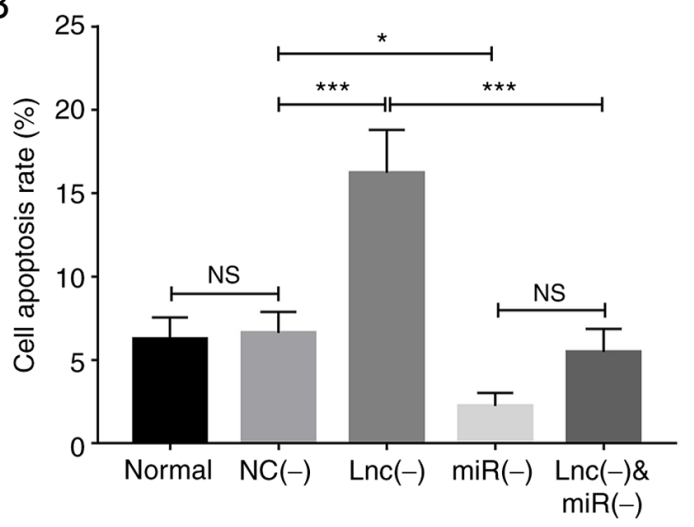

D
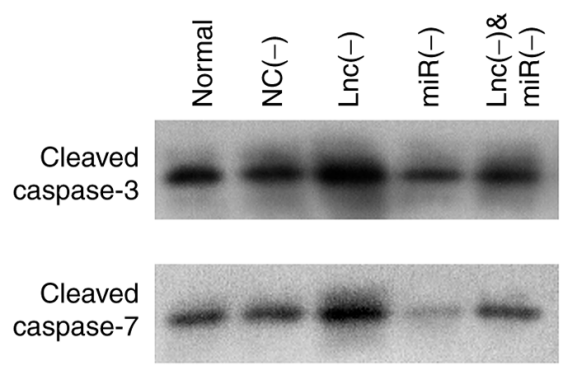

$\beta$-actin

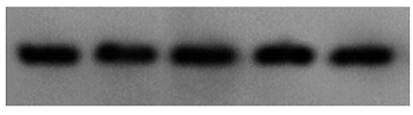

Figure 5. miR-203 inhibitor rescues the effects of IncRNA MEG8 knockdown on the proliferation and apoptosis of hemangioma endothelial cells. (A) Cell proliferation, (B and C) cell apoptosis and (D) cleaved caspase-3 and caspase-7 protein expression levels in the normal, NC (-), lnc (-), miR (-) and lnc (-) + miR (-) groups were analyzed. Statistical differences among groups were determined using a one-way ANOVA followed by a Tukey's post hoc test. All experiments were conducted in triplicate. ${ }^{*} \mathrm{P}<0.05,{ }^{* * *} \mathrm{P}<0.001$. IncRNA, long non-coding RNA; MEG8, maternally expressed 8 , small nucleolar RNA host gene; miR, microRNA; NC, negative control; lnc (-), cells transfected with lncRNA MEG8 small interfering RNA; miR (-), cells transfected with the miR-203 inhibitor; OD, optical density; NS, not significant.

the expression levels of $\operatorname{lncRNA} M E G 8$ are upregulated in HCC tissues and cells and the knockdown of lncRNA MEG8 represses the proliferative, migratory and invasive abilities of NSCLC cells via the $m i R-107 / C D K 6$ signaling axis (9). Another study revealed that in $\mathrm{HCC}$, IncRNA MEG8 regulates the $T G F$ - $\beta$-mediated epigenetic progression of EMT and further promotes EMT-related cell morphological changes and migration in lung and pancreatic cancer cells (10). Furthermore, lncRNA MEG8 has a regulatory role over the Notch signaling pathway in HCC and activation of the Notch signaling pathway serves an important role in the proliferative and involuted phases of hemangioma (16). Regarding the role of $\operatorname{lncRNA}$ $M E G 8$ in hemangioma, only one previous microarray analysis has reported that the expression levels of $1 n c R N A M E G 8$ are upregulated in hemangioma tumor tissues compared with adjacent normal specimens (17). However, to the best of the authors' knowledge, the detailed underlying mechanism of the role of lncRNA MEG8 in hemangioma has not been investigated and was therefore the focus of the current study.

The findings of the present study revealed that $\ln c R N A$ $M E G 8$ knockdown inhibited the proliferation and invasion, but promoted the apoptosis, of HemECs. The results observed in the present study may be due to several different reasons. For example, according to previous studies, lncRNA MEG8 knockdown inhibits adipogenesis via regulating peroxisome proliferator activated receptor (PPAR)- $\alpha$ expression and adipogenic differentiation-related genes (such as $P P A R-\gamma$ ), further suppressing the proliferation and invasion, but enhancing the apoptosis of HemECs $(18,19)$. Furthermore, as lncRNA MEG8 was previously demonstrated to modulate the activation of hepatic stellate cells and the EMT of hepatocytes via the Notch signaling pathway (12), and based on the evidence that Notch ligands were found to be involved in the stimulation of VEGF signaling and angiogenesis in hemangioma (13), IncRNA MEG8 knockdown may inhibit the development and progression of hemangioma via regulating Notch signaling. Finally, $\operatorname{lnc} R N A$ $M E G 8$ knockdown may serve as a competing endogenous RNA of tumor-related miRNAs (such as $m i R-34 a, m i R-200 b$ and $m i R-203)(9,11,20)$, and regulate oncogenic signaling pathways, thereby promoting HemEC proliferation and invasion, but inhibiting apoptosis. However, further investigations are required.

Furthermore, $\ln c R N A M E G 8$ has been found to target several miRNAs (such as $m i R-34 a, m i R-200 b$ and $m i R-203$ ) to promote cellular morphological changes and enhance cell 
A
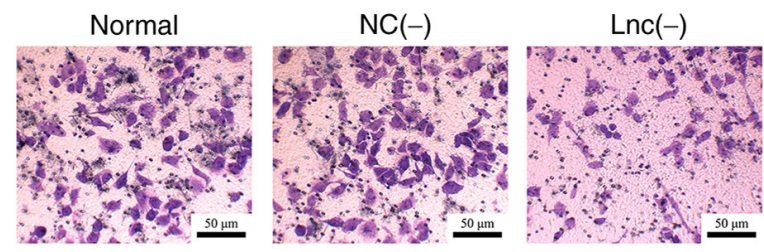

$\operatorname{miR}(-)$

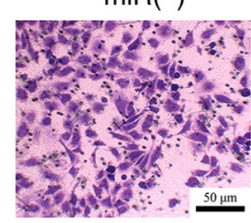

$\operatorname{Lnc}(-) \& \operatorname{miR}(-)$

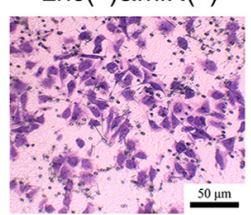

$\mathrm{B}$

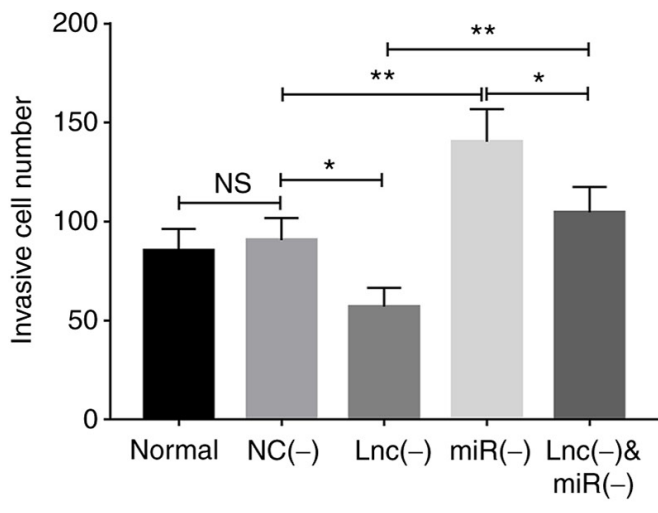

Figure 6. $m i R-203$ inhibitor rescues the effects of lncRNA MEG8 knockdown on the invasion of hemangioma endothelial cells. (A and B) Cell invasion was analyzed in the normal, NC (-), lnc (-), miR (-) and lnc (-) + miR (-) groups. Statistical differences among groups were determined using a one-way ANOVA followed by a Tukey's post hoc test. All experiments were conducted in triplicate. ${ }^{*} \mathrm{P}<0.05,{ }^{* *} \mathrm{P}<0.01$. lncRNA, long non-coding RNA; $M E G 8$, maternally expressed 8, small nucleolar RNA host gene; miR, microRNA; NC, negative control; lnc (-), cells transfected with $l n c R N A M E G 8$ small interfering RNA; miR (-), cells transfected with the miR-203 inhibitor; NS, not significant.
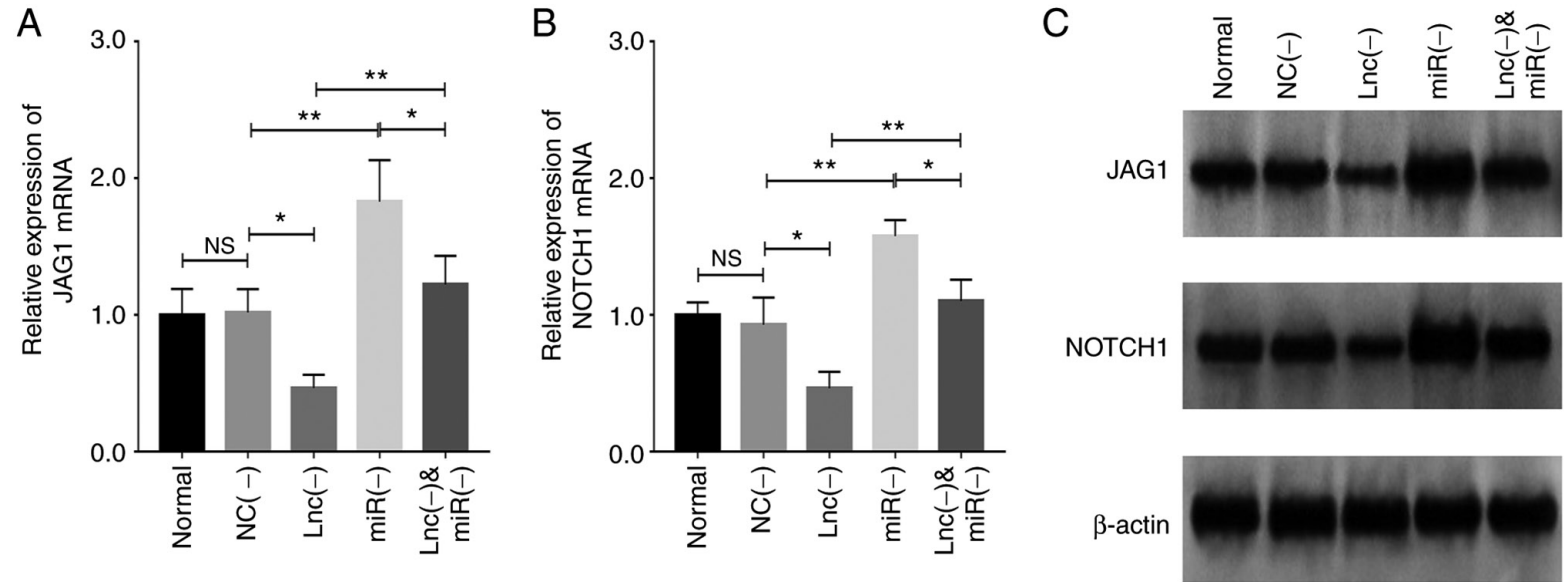

Figure 7. miR-203 inhibitor rescues the effects of lncRNA MEG8 knockdown on the Notch signaling pathway in hemangioma endothelial cells. (A) JAG1 and (B) Notch1 mRNA expression levels were analyzed in the normal, NC (-), lnc (-), miR (-) and lnc (-) + miR (-) groups. (C) Jag1 and Notch1 protein expression levels were analyzed in the normal, NC (-), lnc (-), miR (-) and lnc (-) + miR (-) groups. Statistical differences among groups were determined using a one-way ANOVA followed by a Tukey's post hoc test. All experiments were conducted in triplicate. ${ }^{*} \mathrm{P}<0.05{ }^{* * *} \mathrm{P}<0.01$. lncRNA, long non-coding RNA; miR, microRNA; MEG8, maternally expressed 8, small nucleolar RNA host gene; NC, negative control; lnc (-), cells transfected with lncRNA MEG8 small interfering RNA; miR (-), cells transfected with miR-203 inhibitor; JAG1, jagged canonical notch ligand 1; NS, not significant.

motility in lung and pancreatic cancer (10). The results of the present study were that $\operatorname{lncRNA} M E G 8$ knockdown upregulated $m i R-203$ expression; however, $m i R-203$ silencing did not affect lncRNA MEG8 expression, suggesting that lncRNA $M E G 8$ may be involved in the pathological process of hemangioma via targeting $m i R-203$. Moreover, in the current study, $m i R-203$ silencing could reverse the effects of $1 n c R N A M E G 8$ knockdown on cell proliferation, apoptosis and invasion. According to a previous study, IncRNA MEG 8 knockdown was associated with the recruitment of enhancer of zeste 2 polycomb repressive complex 2 subunit $(E Z H 2)$, which is involved in the transcriptional repression of targeting tumor-suppressor genes and regulated the $E Z H 2$-containing polycomb repressive complex 2 , subsequently upregulating $m i R-203$ expression and EMT activity, which inhibits the progression of hemangioma $(9,21)$. These findings may explain the results obtained in the present study; however, further experimental studies are required for verification.
In addition, it is known that Notch receptors bind with their ligands to activate the Notch signaling pathway and the disrupted expression of core components of the Notch signaling pathway has been found to induce abnormal angiogenesis in hemangioma (16). To determine the effects of $1 n c R N A$ $M E G 8$ and $m i R-203$ on the Notch signaling pathway, experiments were conducted and the results revealed that $\operatorname{lncRNA}$ $M E G 8$ knockdown inactivated the Notch signaling pathway, while miR-203 silencing reversed the inactivating effect of IncRNA MEG8 knockdown on the Notch signaling pathway in HemECs, suggesting that lncRNA MEG8 knockdown inactivated the Notch signaling pathway via targeting $m i R-203$ in hemangioma. As $\ln c R N A M E G 8$ was found to exert a regulatory effect on $T G F-\beta$ expression, which belongs to the growth factor family and is closely associated with the Notch signaling pathway, and considering that $m i R-203$ attenuates the $T G F-\beta$ signaling pathway in breast and ovarian cancer (10), it was hypothesized that $\ln c R N A M E S$ knockdown may regulate 
$T G F-\beta$-associated components and mediate their anti-angiogenic effects via targeting $m i R-203$, thereby inactivating the Notch signaling pathway in hemangioma (10,22-24). However, further experimental studies are required to explore this hypothesis and determine whether this was the mechanism underlying the results of the present study.

However, the present study still has some limitations. Previous evidence indicates that $\operatorname{lncRNA} M E G 8$ is upregulated in infantile hemangioma tissues compared with adjacent non-tumor tissues (17) and other studies observe that IncRNA MEG8 serves as an oncogenic gene in several tumors (9-11) Therefore, the present study did not detect the effect of lncRNA MEG8 overexpression on pro-tumor properties of hemangioma cells. The information might be informative, which needed exploration in further studies.

In conclusion, IncRNA MEG8 knockdown inhibited cell proliferation and invasion, but promoted apoptosis in hemangioma via $m i R$-203-induced mediation of the Notch signaling pathway. This suggested that targeting $\operatorname{lncRNA} M E G 8$ might mediate the $m i R$-203-induced Notch signaling pathway, inhibiting hemangioma progression and providing novel treatment targets for hemangioma.

\section{Acknowledgements}

Not applicable.

\section{Funding}

This study was supported by Program of Hebei Medical Science Research (grant no. 20200582).

\section{Availability of data and materials}

All data generated or analyzed during the present study are included in this published article.

\section{Authors' contributions}

$\mathrm{ZH}$ and $\mathrm{XL}$ contributed to the study design and manuscript writing. ZH, XL, JG, LZ, YC and HY conducted literature research and isolated and cultured hemangioma endothelial cells. ZH and XL contributed to the data acquisition and analysis. The figures are the authors' own work. All authors read and approved the final manuscript. $\mathrm{ZH}$ and XL confirm the authenticity of all the raw data.

\section{Ethics approval and consent to participate}

The present study was approved by the Ethics Committee of Handan Seventh Hospital and written informed consent was obtained from the parents/guardians of each patient prior to participation.

\section{Patient consent for publication}

Not applicable.

\section{Competing interests}

The authors declare that they have no competing interests.

\section{References}

1. DeHart A and Richter G: Hemangioma: Recent advances. F1000Res 8: F1000 Faculty Rev-1926, 2019.

2. Li X, Chen B, Chi D, Zhang Y and Jiang W: lncRNA CASC9 regulates cell migration and invasion in hemangioma endothelial cells by targeting miR-125a-3p/Nrg1. Onco Targets Ther 12: 423-432, 2019.

3. Valdebran M and Wine Lee L: Hemangioma-related syndromes. Curr Opin Pediatr 32: 498-505, 2020.

4. Chi Y, Wang D, Wang J, Yu W and Yang J: Long non-coding RNA in the pathogenesis of cancers. Cells 8: 1015, 2019.

5. Gutschner T and Diederichs S: The hallmarks of cancer: A long non-coding RNA point of view. RNA Biol 9: 703-719, 2012.

6. Lin H, Wang J, Wang T, Wu J, Wang P, Huo X, Zhang J, Pan H and Fan Y: The LncRNA MIR503HG/miR-224-5p/TUSC3 signaling cascade suppresses gastric cancer development via modulating ATF6 Branch of unfolded protein response. Front Oncol 11: 708501, 2021.

7. Cen X, Huang Y, Lu Z, Shao W, Zhuo C, Bao C, Feng S, Wei C, Tang X, Cen L, et al: LncRNA IGFL2-AS1 promotes the proliferation, migration, and invasion of colon cancer cells and is associated with patient prognosis. Cancer Manag Res 13: 5957-5968, 2021.

8. Lou J, Yan W, Li QY, Zhu AK, Tan BQ, Dong R, Zou XZ and Liu T: LncRNA MEG8 plays an oncogenic role in hepatocellular carcinoma progression through miR-367-3p/14-3-3६/TGF $\beta R 1$ axis. Neoplasma 68: 273-282, 2021.

9. Liu Y, Li L, Shang P and Song X: LncRNA MEG8 promotes tumor progression of non-small cell lung cancer via regulating miR-107/CDK6 axis. Anticancer Drugs 31: 1065-1073, 2020.

10. Terashima M, Ishimura A, Wanna-Udom S and Suzuki T: MEG8 long noncoding RNA contributes to epigenetic progression of the epithelial-mesenchymal transition of lung and pancreatic cancer cells. J Biol Chem 293: 18016-18030, 2018.

11. Guo K, Qi D and Huang B: LncRNA MEG8 promotes NSCLC progression by modulating the miR-15a-5p-miR-15b-5p/PSAT1 axis. Cancer Cell Int 21: 84, 2021.

12. Chen T, Lin H, Chen X, Li G, Zhao Y, Zheng L, Shi Z, Zhang K, Hong W and Han T: LncRNA Meg8 suppresses activation of hepatic stellate cells and epithelial-mesenchymal transition of hepatocytes via the Notch pathway. Biochem Biophys Res Commun 521: 921-927, 2020.

13. Ji Y, Chen S, Li K, Li L, Xu C and Xiang B: Signaling pathways in the development of infantile hemangioma. J Hematol Oncol 7: 13, 2014.

14. Khan ZA, Melero-Martin JM, Wu X, Paruchuri S, Boscolo E, Mulliken JB and Bischoff J: Endothelial progenitor cells from infantile hemangioma and umbilical cord blood display unique cellular responses to endostatin. Blood 108: 915-921, 2006.

15. Livak KJ and Schmittgen TD: Analysis of relative gene expression data using real-time quantitative PCR and the 2(-Delta Delta C(T)) method. Methods 25: 402-408, 2001.

16. Zhang H, Wei T, Johnson A, Sun R, Richter G and Strub GM: NOTCH pathway activation in infantile hemangiomas. J Vasc Surg Venous Lymphat Disord 9: 489-496, 2021.

17. Liu X, Lv R, Zhang L, Xu G, Bi J, Gao F, Zhang J, Xue F, Wang F, Wu Y, et al: Long noncoding RNA expression profile of infantile hemangioma identified by microarray analysis. Tumour Biol: Oct 5 , 2016 (Epub ahead of print). doi: 10.1007/s13277-016-5434-y.

18. Zhang B, Dong Y and Zhao Z: LncRNA MEG8 regulates vascular smooth muscle cell proliferation, migration and apoptosis by targeting PPAR $\alpha$. Biochem Biophys Res Commun 510: 171-176, 2019.

19. Yuan SM, Guo Y, Xu Y, Wang M, Chen HN and Shen WM: The adipogenesis in infantile hemangioma and the expression of adipogenic-related genes. Int J Clin Exp Pathol 10: 11596-11602, 2017.

20. Fang Y and Fullwood MJ: Roles, functions, and mechanisms of long non-coding RNAs in cancer. Genomics Proteomics Bioinformatics 14: 42-54, 2016.

21. Eich ML,Athar M,Ferguson JE III and Varambally S: EZH2-targeted therapies in cancer: Hype or a reality. Cancer Res 80: 5449-5458, 2020.

22. Carmeliet P and Jain RK: Molecular mechanisms and clinical applications of angiogenesis. Nature 473: 298-307, 2011.

23. He S, Zhang G, Dong H, Ma M and Sun Q: MiR-203 facilitates tumor growth and metastasis by targeting fibroblast growth factor 2 in breast cancer. Onco Targets Ther 9: 6203-6210, 2016

24. Wang B, Li X, Zhao G, Yan H, Dong P, Watari H, Sims M, Li W, Pfeffer LM, Guo Y and Yue J: MiR-203 inhibits ovarian tumor metastasis by targeting BIRC5 and attenuating the TGF $\beta$ pathway. J Exp Clin Cancer Res 37: 235, 2018. 